\title{
Stability Analysis of a fluid in Horizontal and Inclined Channels
}

\section{Ala'a Abdul-raheem Ahmed Hammodat \\ College of Education \\ University of Mosul \\ Received on : $23 / 9 / 2013$}

\section{ABSTRACT}

\section{Accepted on: 12/2/2014}

This paper is devoted to analysis the stability of horizontal and inclination in a glazing cavities of equations that we expose to disturbance. This analysis is done by finding the eigenvalues of the system which enable us to investigate the grow of disturbance after setting glazing cavities equations in linearization form. We obtain from our results that the equations are stable when the real part of wave velocity is negative, and unstable when it is positive .

Keywords: Stability, Reynold Number, Wave Number, Rayleigh Number.

$$
\text { علاء عبدالرحيم احد حمود/ت الاستقرية لمائع في التجاويف الزجاجية الأفقية والمائلة }
$$

كلية التربية، جامعة الموصل

تاريخ قبول البحث: 2014/2/12

تاريخ استلام البحث: 2013/9/23

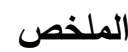

هذا البحث مكرس لتحليل الاستقرارية من النوع الأفقي والمائل لمنظومة من المعادلات عرضناها لاضطراب

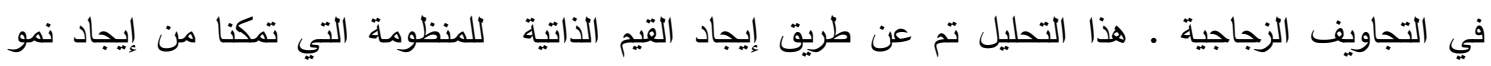

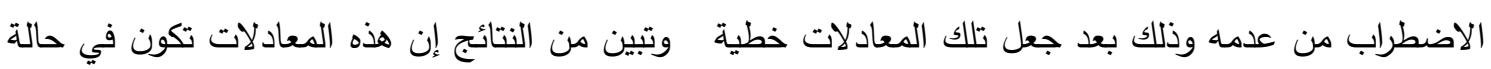
استقرار عندما يكون الجزء الحقيقي لسرعة الموجة كمية سالبة وفي حالة عدم استقرار عندما تكون هذه الكمية

$$
\text { 1 الموجبة . الكلمات المفتاحية: الاستقرارية ، عدد رينولد ، العدد الموجي ، عدد رايلي. }
$$

يعد ميكانيك الموائع إحدى العلوم الهندية التي تكون أساسيات العلوم الهندسية الأخرى كلها , وهذا العلم

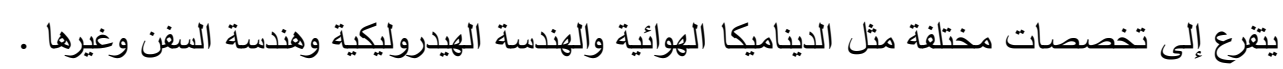

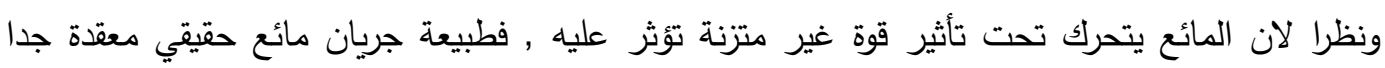

حيث إن القوانين الأساسية التي تصف الحركة الكاملة لمائع لم توضع بطريقة سهلة والحلول الرياضية تحتاج إلى تئى

$$
\text { الاستعانة بالتجارب العملية . }
$$

إن دراسة حركة المائع تقودنا إلى دراسة استقرارية هذا المائع , فإذا تعرض مائع جاري إلى إزعاج فقد يزول

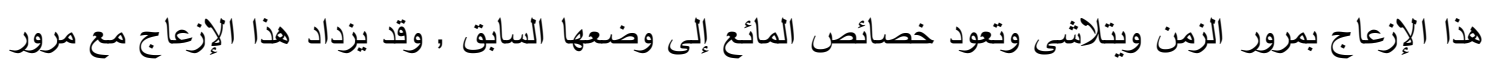

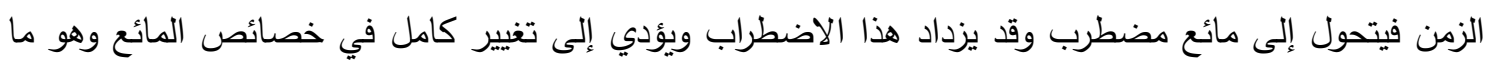
يسمى بالفوضوية .

يعد نظام التجويف الزجاجي (Glazing Cavity System) احد أهم مكونات منتجات نسق النوافذ والأبواب

في مبنى , اذ يتكون عادة من لوحين زجاجيين (Glass Panes) بينهما مادة بينية (Spacer) , توضع هذه المادة

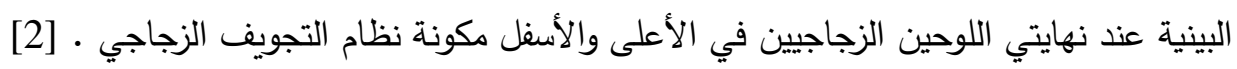




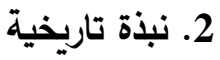

لقد أجريت دراسات عديدة حول موضوع الاستقرارية وانتقال الحرارة في التجاويف الزجاجية وفيما يلي بعض الأعمال السابقة في هذا المجال :

ناقش [8] مسالة الحمل الحراري الطبيعي لمائع محتوى في تجويف طويل مغلق أفقي لمقطع مستطيل الثكل

محدد بجدارين احدهما ساخن والأخر بارد , وقد تم حل معادلتي الحرارة والحركة عدديا كون السائل دائرا. درس [4] عدد رايلي (Rayleigh) الحرج بالاتجاه العمودي والمائل باستخدام تجويف ذو دول مقطع نسبي 44 (Aspect ratio)

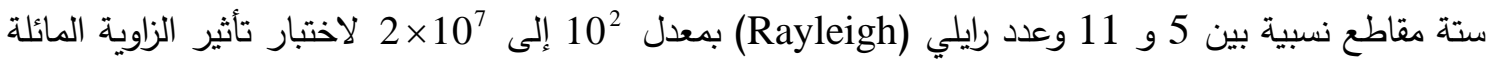
والمقطع النسبي في معدل انتقال الحرارة وطور العلاقة عند الزاوية المائلة $60^{\circ}$ وقد وجد أيضا اندراجا خطيا بين بين الزاويتين 60 و 90 90 ـ ناقش [5] الحل التحليلي باستخدام المحاكاة العددية ووجد أن الحل الملائم لثق عمودي يكون عدد رايلي (Rayleigh) كبيرا ، ودرس [7] التجاويف ذات المقاطع النسبية 1 و 3 و 4 وبينت النتائج لهذه المقاطع النسبية أن نقطة الانتقال لنمط الجريان يذهب إلى زاوية كبيرة من الميلان عندما يزداد عداد عدد رايلي (Rayleigh) قابلية أعلى لعزل المحيط الخارجي عن المحيط الداخلي . لدارسه

يتكون النموذج الرياضي من جدارين عموديين موصليين للحرارة اللذين يمثلان الطبقة الداخلية للوحين الزجاجيين (Glass Panes) اللذين يكونان التجويف الزجاجي , احد هذين الجدارين يكون حار ونرمز لدرجة حرارته

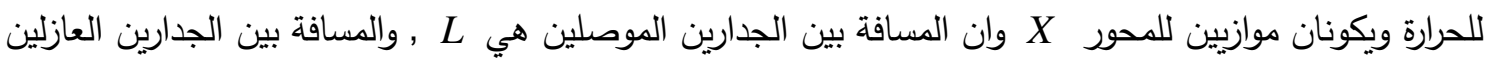
هي H ـ ويملا هذا التجويف بمائع غير قابل للانضغاط ومع وجود التشتت في اللزوجة. وكما مبين في الثكل (1)

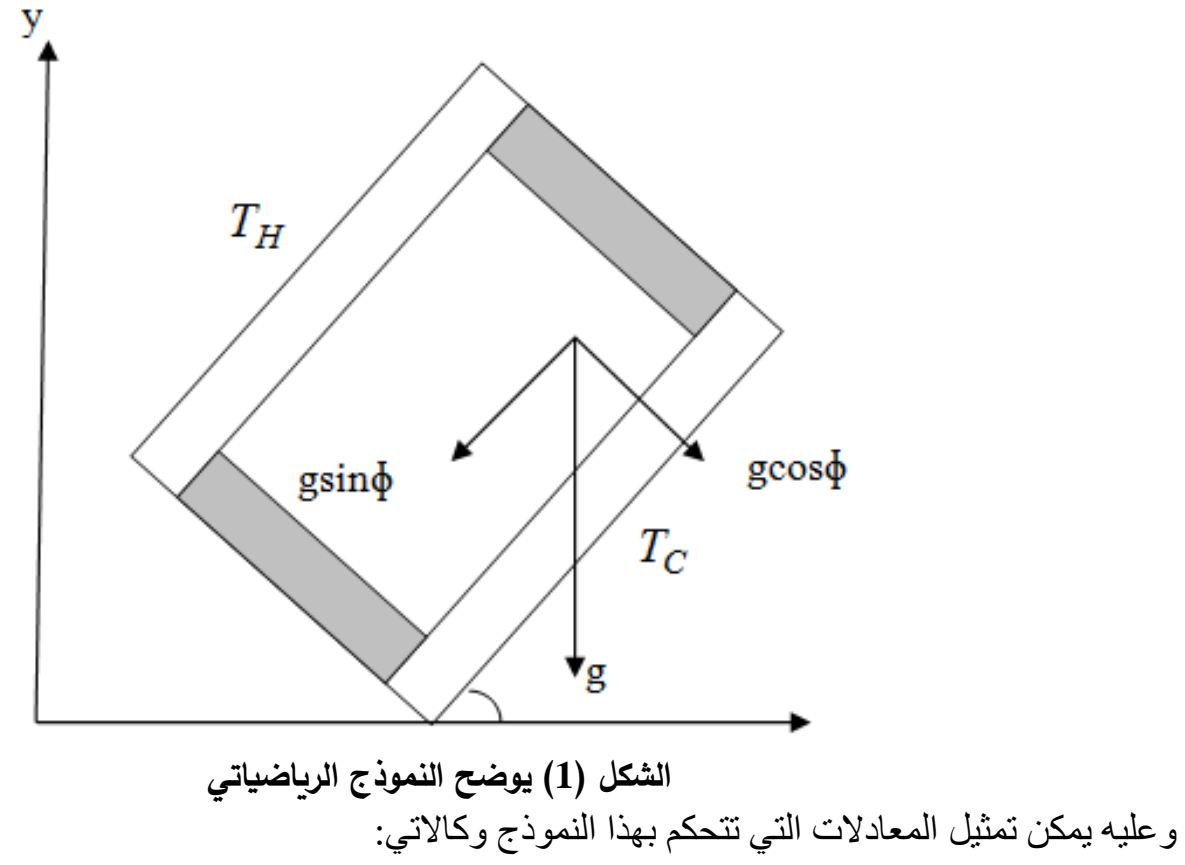


$\frac{\partial u}{\partial x}+\frac{\partial v}{\partial y}=0$

$\frac{\partial u}{\partial t}+u \frac{\partial u}{\partial x}+v \frac{\partial u}{\partial y}=-\frac{1}{\rho} \frac{\partial P}{\partial x}+v \nabla^{2} u-\beta g\left(T-T_{0}\right) \cos \varphi$.

$\frac{\partial v}{\partial t}+u \frac{\partial v}{\partial x}+v \frac{\partial v}{\partial y}=-\frac{1}{\rho} \frac{\partial P}{\partial y}+v \nabla^{2} v+\beta g\left(T-T_{0}\right) \sin \varphi$

$\frac{\partial T}{\partial t}+u \frac{\partial T}{\partial x}+v \frac{\partial T}{\partial y}=\frac{k}{\rho C_{p}} \nabla^{2} T+\varepsilon\left[\left(\frac{\partial u}{\partial y}\right)^{2}+\left(\frac{\partial v}{\partial x}\right)^{2}\right]$.

حيث أن

$T(0, y)=T_{H}$

$T(L, y)=T_{C}$

$\left.\frac{\partial T}{\partial y}\right|_{y=0}=0$

و عند السطحين العلوي و السفلي هي:

$\left.\frac{\partial T}{\partial y}\right|_{y=H}=0$

$u(0, y)=v(0, y)=0$

بينما الشروط الحدودية للسر عة تكون:

$u(L, y)=v(L, y)=0$

$u(x, 0)=v(x, 0)=0$

$u(x, H)=v(x, H)=0$

4. التحليل البعدي (Dimensional Analysis):

لغرض إيجاد المعادلات اللابعدية للمعادلات (3.1) , (3.2) , (3.3) و (3.4) سوف نفرض بعض القيم

[9]: اللابعدية الترن

$u^{*}=\frac{u}{U}$

$v^{*}=\frac{v}{V}$

$U=\frac{\alpha}{L} \sqrt{R a \operatorname{Pr}}=\sqrt{g \beta \Delta T L}$

$R a=\frac{\rho g \beta\left(T_{1}-T_{0}\right) L^{3}}{\mu \alpha}$

$x^{*}=\frac{x}{L}$

$y^{*}=\frac{y}{L}$

$\theta=\frac{T-T_{0}}{T_{1}-T_{0}}$

$\operatorname{Pr}=\frac{v}{\alpha}=\frac{\mu C_{p}}{k}$

$t^{*}=\frac{t U}{L}$

$q=T_{1}^{4} \sigma Q$

$P=P * \rho U^{2}$

وبتعويض هذه القيم في المعادلات (3.1) , (3.2) , (3.3) و (3.4) نحصل على المعادلات اللابعدية 
$\frac{\partial u^{*}}{\partial x^{*}}+\frac{\partial v^{*}}{\partial y^{*}}=0$

$\frac{\partial u^{*}}{\partial t^{*}}+u * \frac{\partial u^{*}}{\partial x^{*}}+v^{*} \frac{\partial u^{*}}{\partial y^{*}}=-\frac{\partial p^{*}}{\partial x^{*}}+\frac{1}{\operatorname{Re}}\left[\frac{\partial^{2} u^{*}}{\partial x^{2}}+\frac{\partial^{2} u^{*}}{\partial y^{2}}\right]-(E-H \theta) \cos \varphi$

$\frac{\partial v^{*}}{\partial t^{*}}+u * \frac{\partial v^{*}}{\partial x^{*}}+v^{*} \frac{\partial v^{*}}{\partial y^{*}}=-\frac{\partial p^{*}}{\partial y^{*}}+\frac{1}{\operatorname{Re}}\left[\frac{\partial^{2} v^{*}}{\partial x^{*}}+\frac{\partial^{2} v^{*}}{\partial y^{* 2}}\right]+(E-H \theta) \sin \varphi$.

$\sqrt{R a \operatorname{Pr}}\left[\frac{\partial \theta}{\partial t^{*}}+u^{*} \frac{\partial \theta}{\partial x^{*}}+v^{*} \frac{\partial \theta}{\partial y^{*}}\right]=\left[\frac{\partial^{2} \theta}{\partial x^{2}}+\frac{\partial^{2} \theta}{\partial y^{2}}\right]+\operatorname{Pr} E c\left[\left(\frac{\partial u *}{\partial y^{*}}\right)^{2}+\left(\frac{\partial v^{*}}{\partial x *}\right)^{2}\right]$

$\theta\left(0, y^{*}\right)=\theta_{H}$

و الثروط الحدودية في الحالة اللابعدية تصبح بالثكل التالي :

$\theta\left(L, y^{*}\right)=\theta_{C}$

$\left.\frac{\partial \theta}{\partial y *}\right|_{y^{*}=0}=0$ and $\left.\frac{\partial \theta}{\partial y^{*}}\right|_{y^{*}=H}=0$

$u\left(0, y^{*}\right)=v\left(0, y^{*}\right)=0$

$u\left(L, y^{*}\right)=v\left(L, y^{*}\right)=0$

$u\left(x^{*}, 0\right)=v\left(x^{*}, H\right)=0$

$u\left(x^{*}, H\right)=v\left(x^{*}, H\right)=0$

5. تحليل الاستقرارية (Stability Analysis)

لغـرض تحليـل الاسـتقرارية للمعـادلات (4.1) , (4.2) , (4.3) و (4.4) نجـز أ كـلا مـن

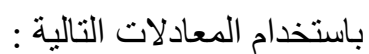

$u *(x, y, t)=u_{1} *(x, y)+u_{2} *(x, y, t)$

$v^{*}(x, y, t)=v_{1} *(x, y)+v_{2} *(x, y, t)$

$p^{*}(x, y, t)=p_{1} *(x, y)+p_{2} *(x, y, t)$

$\theta(x, y, t)=\theta_{1}(x, y)+\theta_{2}(x, y, t)$

حيث ان

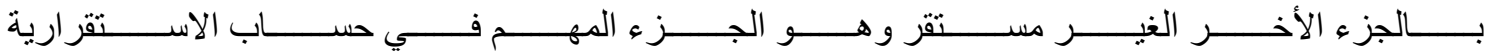
. [1] $u_{2} *(x, y), v_{2} *(x, y), p_{2} *(x, y), \theta_{2}(x, y)$

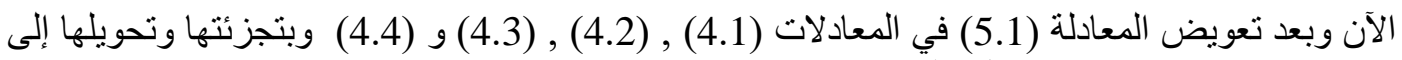

حالتي الاستقر ارية والاضطر اب نحصل على :

$\frac{\partial u_{2} *}{\partial x^{*}}+\frac{\partial v_{2} *}{\partial y *}=0$

$\frac{\partial u_{2} *}{\partial t *}+u_{1} * \frac{\partial u_{2} *}{\partial x *}+u_{2} * \frac{\partial u_{1} *}{\partial x *}+u_{2} * \frac{\partial u_{2} *}{\partial x *}+v_{1} * \frac{\partial u_{2} *}{\partial y *}+v_{2} * \frac{\partial u_{1} *}{\partial y *}+v_{2} * \frac{\partial u_{2} *}{\partial y *}$

$=-\frac{\partial p_{2} *}{\partial x^{*}}+\frac{1}{\operatorname{Re}}\left[\frac{\partial^{2} u_{2} *}{\partial x^{2}}+\frac{\partial^{2} u_{2} *}{\partial *^{2}}\right]+H \theta_{2} \cos \varphi$ 


$$
\begin{aligned}
& \frac{\partial v_{2} *}{\partial t *}+u_{1} * \frac{\partial v_{2} *}{\partial x *}+u_{2} * \frac{\partial v_{1} *}{\partial x *}+u_{2} * \frac{\partial v_{2} *}{\partial x *}+v_{2} * \frac{\partial v_{1} *}{\partial y *}+v_{1} * \frac{\partial v_{2} *}{\partial y *}+v_{2} * \frac{\partial v_{2} *}{\partial y *} \\
& =-\frac{\partial p_{2} *}{\partial y *}+\frac{1}{\operatorname{Re}}\left[\frac{\partial^{2} v_{2} *}{\partial x *^{2}}+\frac{\partial^{2} v_{2} *}{\partial *^{2}}\right]-H \theta_{2} \sin \varphi
\end{aligned} .
$$

$\sqrt{R a \operatorname{Pr}}\left[\frac{\partial \theta_{2}}{\partial t *}+u_{1} * \frac{\partial \theta_{2}}{\partial x *}+u_{2} * \frac{\partial \theta_{1}}{\partial x *}+u_{2} * \frac{\partial \theta_{2}}{\partial x *}+v_{1} * \frac{\partial \theta_{2}}{\partial y *}+v_{2} * \frac{\partial \theta_{1}}{\partial y *}+v_{2} * \frac{\partial \theta_{2}}{\partial y *}\right]$

$=\frac{\partial^{2} \theta_{2}}{\partial x^{2}}+\frac{\partial^{2} \theta_{2}}{\partial *^{2}}+\operatorname{Pr} E c\left[\left(\frac{\partial u_{2} *}{\partial y *}\right)^{2}+\left(\frac{\partial v_{2} *}{\partial x^{2}}\right)^{2}\right]$

ولتحويل المعـادلات (5.1), (5.2) , (5.3) و (5.4) إلى معـادلات خطيـة نقوم بحذف الحدود اللاخطيـة منهـا

$\frac{\partial u_{2}^{*}}{\partial x^{*}}+\frac{\partial v_{2}^{*}}{\partial y^{*}}=0$

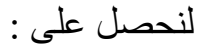

$\frac{\partial u_{2} *}{\partial t^{*}}=-\frac{\partial p_{2} *}{\partial x^{*}}+\frac{1}{\operatorname{Re}}\left[\frac{\partial^{2} u_{2} *}{\partial x^{2}}+\frac{\partial^{2} u_{2} *}{\partial *^{2}}\right]+H \theta_{2} \cos \varphi$.

$\frac{\partial v_{2} *}{\partial t^{*}}=-\frac{\partial p_{2} *}{\partial y^{*}}+\frac{1}{\operatorname{Re}}\left[\frac{\partial^{2} v_{2}^{*}}{\partial x^{2}}+\frac{\partial^{2} v_{2} *}{\partial *^{2}}\right]-H \theta_{2} \sin \varphi$.

$\sqrt{R a \operatorname{Pr}}\left[\frac{\partial \theta_{2}}{\partial t *}\right]=\frac{\partial^{2} \theta_{2}}{\partial x^{2}}+\frac{\partial^{2} \theta_{2}}{\partial y *^{2}}+\operatorname{Pr} E c\left[\left(\frac{\partial u_{2} *}{\partial y *}\right)^{2}+\left(\frac{\partial v_{2} *}{\partial x^{2}}\right)^{2}\right]$.

6.

لإيجـاد الحـل للمعـادلات (5.6) , (5.7) , (5.8) و (5.9) , نتصـور أن الاضـطر اب حاصـل بالاتجـاهين

x $x, y$

$$
\begin{aligned}
& u_{2} *=u(y) e^{i k x+a t} \\
& v_{2} *=v(y) e^{i k x+a t} \\
& p_{2}^{*}=p(y) e^{i k x+a t} \\
& \theta_{2}=\theta(y) e^{i k x+a t}
\end{aligned}
$$

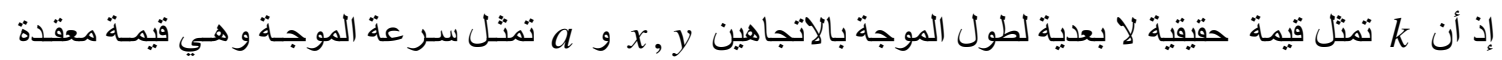

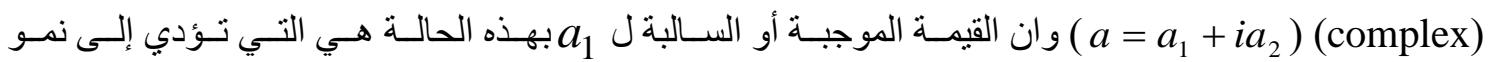
الاضطر اب أو تلاشيه على التو الي ـ فعندما تكون تكون

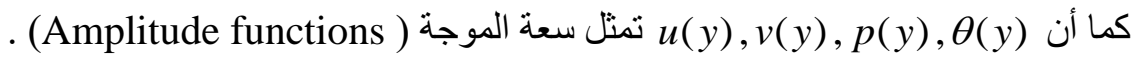
و بتعويض (6.1) في المعادلات (5.6) , (5.7) , (5.8) و (5.9) ومع فرض التالبة : 


$$
\begin{aligned}
& u^{\prime}=h \\
& h^{\prime}=A u+B p-C \theta \\
& p^{\prime}=-B h-Z v \\
& v^{\prime}=-E u \\
& \theta^{\prime}=s \\
& s^{\prime}=F \theta-G u-M p+J v
\end{aligned}
$$

حيث ان

$A=a \operatorname{Re}+K^{2} \quad, \quad B=i k \operatorname{Re} \quad, \quad C=-H \operatorname{Re} \quad, \quad Z=-\left(a+\frac{k^{2}}{\operatorname{Re}}\right) \quad, \quad E=-\frac{i k}{\operatorname{Re}}$ $F=a \sqrt{R a \operatorname{Pr}}+k^{2}+\operatorname{Pr} E c H \operatorname{Re} \quad, \quad G=-\operatorname{Pr} E c\left(a \operatorname{Re}+k^{2}\right) \quad, \quad M=-i k \operatorname{Pr} E c \operatorname{Re}$ $J=\operatorname{Pr} E c k^{2}$

وبايجاد القيم الذاتية للمنظومة (6.2) نحصل على المعادلة التالية : $f(\lambda)=\lambda^{6}+\left(B^{2}-F-A\right) \lambda^{4}+\left(A F-C G-B^{2} F+B C M-E Z B\right) \lambda^{2}+(E J C) \lambda+\ldots$ $\ldots+(Z E C M-Z E B F)=0$

بينما عندما تكون $\varphi=45^{\circ}$

$$
\begin{aligned}
& u^{\prime}=h \\
& h^{\prime}=A u+B p-C \theta \\
& p^{\prime}=-B h-Z v-\left(\frac{H}{\sqrt{2}}\right) \theta \\
& v^{\prime}=-E u \\
& \theta^{\prime}=s \\
& s^{\prime}=F \theta-G u-M p+J v
\end{aligned}
$$

$A=a \operatorname{Re}+k^{2} \quad, \quad B=i k \operatorname{Re} \quad, \quad C=-\frac{H \operatorname{Re}}{\sqrt{2}} \quad, \quad Z=-\left(a+\frac{k^{2}}{\operatorname{Re}}\right) \quad, \quad E=-\frac{i k}{\operatorname{Re}}$, $W=\frac{H}{\sqrt{2}} \quad, \quad F=a \sqrt{R a \operatorname{Pr}}+k^{2}+\frac{\operatorname{Pr} E c H \operatorname{Re}}{\sqrt{2}} \quad, \quad G=-\operatorname{Pr} E c\left(a \operatorname{Re}+k^{2}\right)$, $M=i k \operatorname{Re} \operatorname{Pr} E c \quad, \quad J=\operatorname{Pr} E c k^{2}$

وبإيجاد القيم الذاتية للمنظومة (6.4) نحصل على المعادلة التالية : $f(\lambda)=\lambda^{6}+\left(B^{2}-F-A\right) \lambda^{4}+(-W M) \lambda^{3}+\left(C B M+A F-B^{2} F-B E Z-C G\right) \lambda^{2}+\ldots$

$\ldots+(A M W-C E J-B G W) \lambda+(B E Z F-C E Z M-B E J W)=0$

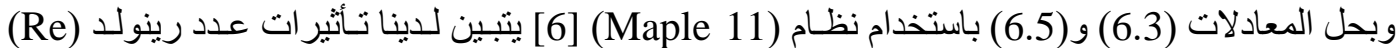

و عدد بر انتدل (Pr) و عدد رايلي (Ra) بالإضافة إلى نأثنير المبلان وكما مبين في الأشكال (10.3 (10,9,8,7,6,5,4,3,2) 
(Conclusions)

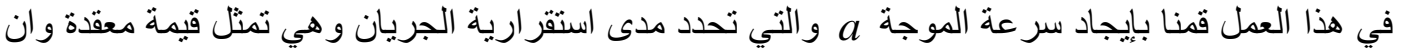

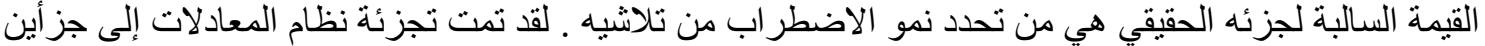

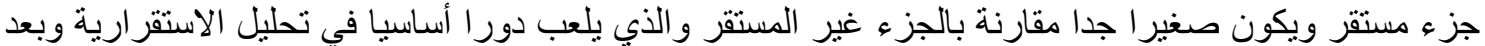

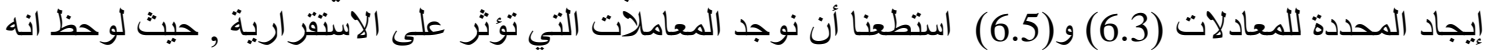

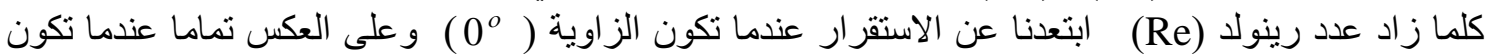

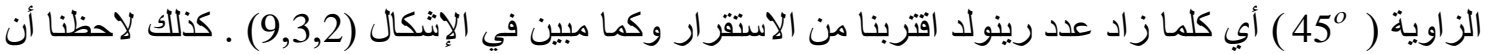

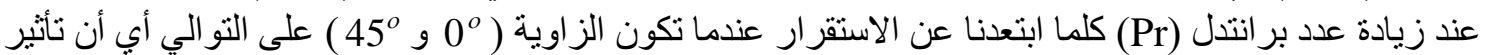
الميلان يكون طفيفا وكما مبين في الأشكال (8,5,4) ابتعدنا عن الاستقرار في الحالتين ( $0^{\circ}$ و $45^{\circ}$ ) وكما مبين في الأشكال (10,7,6) وكال

(3) (3) (20) (20)
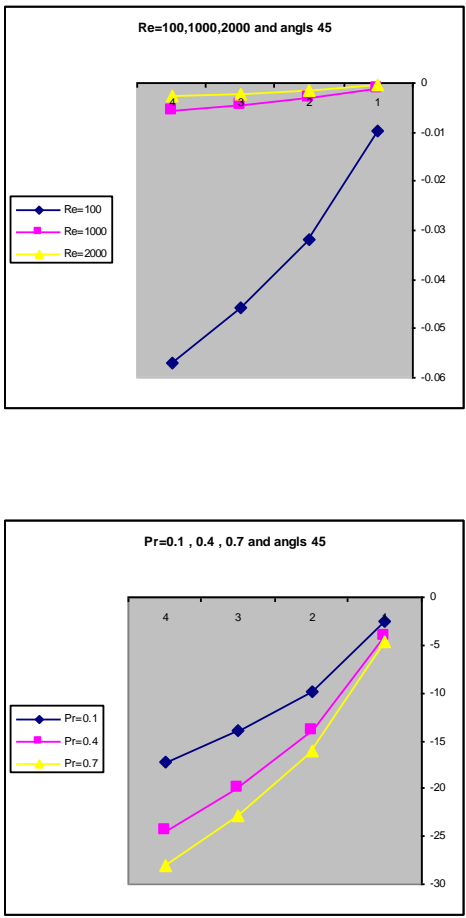

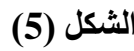

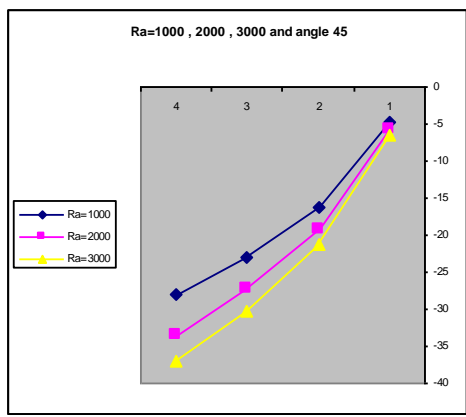

الثكل (7)

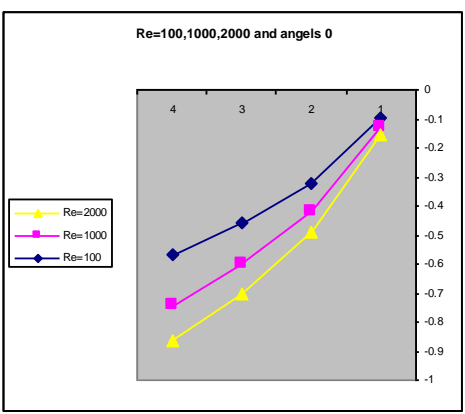

الثكل (2)

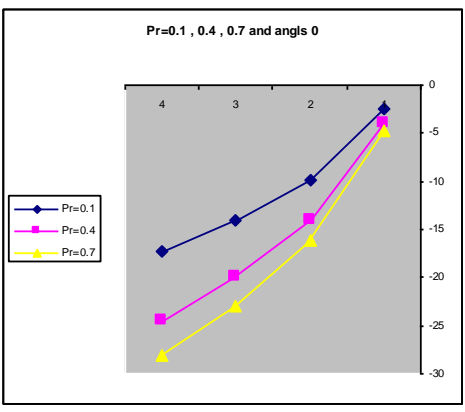

الثكل (4)

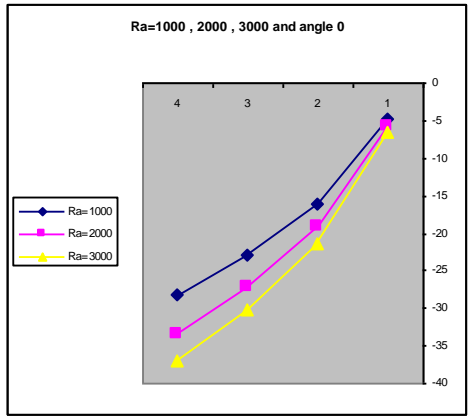

الثكل (6) 


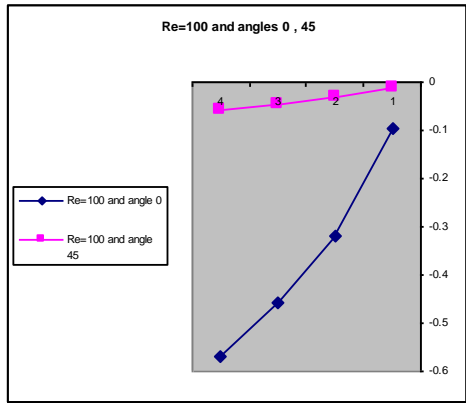

(9) الشكل
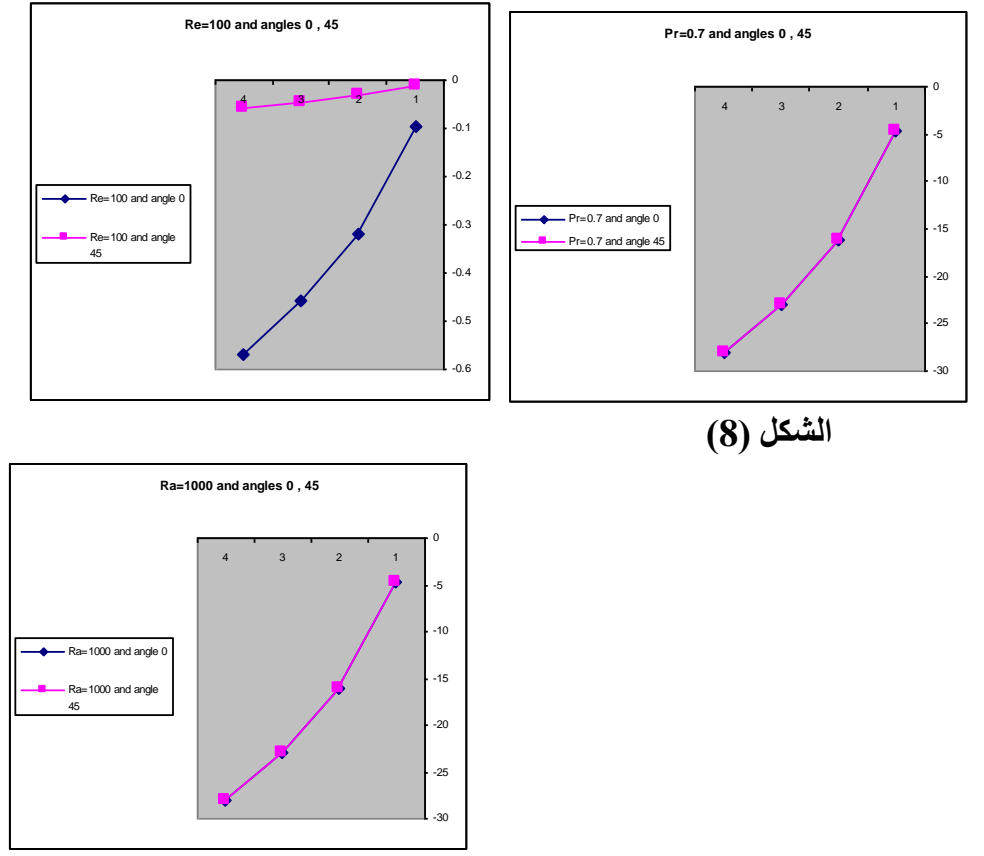

الشكل (8)

الثكل (10) 


\section{المصادر}

[1] Ala'a A.H. \& Ahmed M. J. ,(2012), " A theoretical study of stability of flowing and heat transfer systems in vessels and channels ", Ph.D. Thesis , Mosul university.

[2] Ahmed M.J. \& Osama T. ,(2010), " heat transfer in a glazing cavities " , M.Sc. Thesis, Mosul university .

[3] Elsherbiny S.M. , Raithby G.D. \& Hollands k.G. ,(1982)," heat transfer by natural convection across vertical and inlined Air layers " ,transactions of the ASME, Vol.104, p.p 96-102 .

[4] Hollands K.G.T. \& Konicek L. ,(1973), " Experimental study of the stability of differentially heated inclined Air layers " Int. J. of heat and mass transfer, Vol.16, p.p 1467-1476.

[5] Kimura S. \& Bejan A. ,(1984), " The boundary layer natural convection Regimes in rectangular cavity with uniform heat flux from the side ", J. of heat transfer, Vol. 106 , p.p 98-102 .

[6] Monagan M.B. ,( 2003)," Maple 11 Advanced programming guide " .

[7] Soong C.Y., Tzeng P.Y., Chiang D. C. \& Sheu T.S. ,(1996)," Numerical study on Mode-transition on natural convection in differentially heated inclined enclosure " Int. J. of Heat and Mass Transfer, Vol. 39 , No. 14 , pp. 2869-2882 .

[8] Wilkes J.O. \& Churchil S.W. ,(1966), " The finite difference computation of natural convection in a rectangular enclosure " A.I.che Journal, Vol. 12 , No. 1 , p.p 161-167 .

[9] Yang Y. ,(2003)," Natural convection flow and heat transfer in vertical and Inclined glazing cavities " , M.Sc. Thesis, University of Massachusetts. 